\title{
Nonradiative decay of the lowest excited singlet state of 2-aminopyridine is considerably faster than the radiative decay
}

\author{
Feng Zhang, ${ }^{1,2}$ Yue-Jie Ai, ${ }^{1,2}$ Yi Luo, $^{2, a)}$ and Wei-Hai Fang ${ }^{1}$ \\ ${ }^{1}$ College of Chemistry, Beijing Normal University, Beijing 100875, China \\ ${ }^{2}$ Theoretical Chemistry, School of Biotechnology, Royal Institute of Technology, S-10691 Stockholm, Sweden
}

(Received 4 January 2009; accepted 12 March 2009; published online 14 April 2009)

\begin{abstract}
$A b$ initio calculations reveal that radiative lifetime of the lowest excited singlet state of 2-aminopyridine molecule should be around $20 \mathrm{~ns}$, consistent with the molecules of the same type but is about one order of magnitude larger than the claimed experimental fluorescent lifetime in recent years. An $S_{1} / S_{0}$ conical intersection close to the $S_{1}$ state has been located, which could be the possible nonradiative channel that is responsible for the fast decay observed in the experiment. (C) 2009 American Institute of Physics. [DOI: 10.1063/1.3113664]
\end{abstract}

\section{INTRODUCTION}

A simple molecule such as 2-aminopyridine (2-AP) becomes interesting probably mainly because its hydrogenbonded dimer has been used as a model system to mimic the DNA base pairs. ${ }^{1,2}$ It is well known that DNA can be easily damaged by the ultraviolet component (wavelength $<400 \mathrm{~nm}$ ) of the sunlight. The possible mechanisms for photodamage and photostability of DNA bases have been widely studied. The ultrashort lifetime of DNA may be associated with base-pairing and base-stacking interaction, genuine electronic property of DNA bases, and the solvation. ${ }^{1-10}$ However, it is difficult to identify the exact mechanism due to the complexity of DNA structures. In this context, the 2-AP dimer was used as a simplified model for DNA base pairs by Schultz et al. ${ }^{1}$ and Sobolewski and Domcke. ${ }^{2}$ In their pump-probe ionization spectroscopy studies, it was found that the excited-state population of the isolated 2-AP monomer relaxed with a lifetime of $\tau=1.5 \mathrm{~ns}$, while the 2-AP dimer showed a quite reduced lifetime of $\tau=65 \mathrm{ps}$. Based on the high-resolution electronic spectra of 2-AP molecule in gas phase, Borst et al. ${ }^{11}$ concluded that the measured fluorescence lifetime of 2-AP monomer was about $1.5 \mathrm{~ns}$. With this in mind, the reduced lifetime of the dimer could thus be considered as the result of the involvement of nonradiative decay due to single proton transferring between two monomers. ${ }^{1}$

Early in 1976, Babiak and Testa ${ }^{12}$ studied the fluorescence yields of 2-, 3-, and 4-aminopyridines in a variety of solvents and concluded that the fluorescence spectra of 2and 3-AP have very similar behavior. Indeed, the experimental fluorescence lifetime was found to be $21.2 \pm 0.1 \mathrm{~ns}$ (Ref. 12) for 3-AP in neutral form, which was close to the value of $18 \mathrm{~ns}$ (Ref. 13) for the natural 2-AP. For the latter, values in the region of 10-20 ns (Ref. 14) were also reported. However, in a supersonic beam study by Hager $e t$ al.,${ }^{15}$ the upper limit on the fluorescence of the bare 2-AP molecule was set to be $2 \mathrm{~ns}$, a view that seems to be supported by recent experimental results. ${ }^{1,11}$ It is noted that the difference in fluo-

${ }^{\text {a)} E l e c t r o n i c ~ m a i l: ~ l u o @ t h e o c h e m . k t h . s e . ~}$ rescence lifetimes of 2-AP in gas phase and in solution is quite unusual, which might be explained by collisions between solvent and solute molecules, which accelerate the intrastate vibrational relaxation to the minimum of the $S_{1}$ state and consequently reduce the probability of nonradiative decays.

Many experimental and theoretical studies have been devoted to photophysical properties of molecules with structures similar to 2-AP. ${ }^{9,10,16-21}$ The surface intersection between the $S_{1}$ and $S_{0}$ states was found to play a crucial role for radiationless deactivation process. An extremely facile internal conversion has been identified for all nucleobases because of their genuine molecular property. Femtosecond transient absorption spectroscopy and ab initio calculations for excited-state deactivation of pyridine molecule led to the conclusion that along a "prefulvenic" pathway, there was an ultrashort internal conversion between the $S_{1}$ state and the ground state. ${ }^{20}$ Recently, an out-of-plane ring deformation was confirmed to be a deactivation pathway for the 6-AP molecule in the $S_{1}$ state. $^{21}$ To resolve the uncertainty related to the lifetime of $S_{1}$ state of 2-AP molecule, we have carried out $a b$ initio calculations to identify the contributions from possible radiative and nonradiative channels. To the best of our knowledge, there is no similar study reported in the literature.

\section{COMPUTATIONAL DETAILS}

All geometry optimizations were carried out by using the complete active space self-consistent filed method (CASSCF) and the cc-pVDZ basis set without any symmetry constrained. The active space used for the optimization of stationary points (local minima and saddle points) on both ground $\left(S_{0}\right)$ and the lowest singlet excited $\left(S_{1}\right)$ states included ten electrons distributed over eight valence orbits, denoted as $\operatorname{CAS}(10,8)$. To study the ring deformation on the lowest ${ }^{1} \pi \pi^{*}$ state, the active space is composed of eight conjugated $\pi$ orbits $\left(4 \pi\right.$ and $\left.4 \pi^{*}\right)$ in the aromatic ring and the $\mathrm{NH}_{2}$ group. To construct the energy profile of the $\mathrm{N}-\mathrm{H}$ dissociation on the ${ }^{1} \pi \sigma^{*}$ state, the CAS $(10,8)$ space was constituted with six conjugated $\pi$ orbitals $\left(3 \pi\right.$ and $\left.3 \pi^{*}\right)$ on the 
ring and a pair of the $\mathrm{N}-\mathrm{H} \sigma$ and $\sigma^{*}$ orbits. Nature of the stationary and saddle points was validated by Hessian calculations. Zero point energy correction has been taken into account for the barrier heights and dissociation energies. All calculations were carried out using GAUSSIAN03 program package. $^{22}$

The radiative lifetime can be calculated by a simple working formula $\tau=1.5003 / f \Delta E^{2}$, where $f$ is the oscillator strength of the excited state, $\Delta E$ is the emission energy in $\mathrm{cm}^{-1}$. Linear response (LR) calculations at multiconfiguration self-consisted field (MCSCF), couple cluster singles and doubles (CCSD) models, and density functional theory levels as implemented in DALTON 2.0 program ${ }^{23}$ were performed to obtain the values of $f$ and $\Delta E$. The active space of eight orbits occupied with ten electrons was also used in the LR MCSCF calculation to be consistent with the state-specified CASSCF calculation. The Becke-3-Lee-Yang-Parr (B3LYP) functional was chosen to perform the DFT-LR calculation as implemented in DALTON 2.0. ${ }^{23}$ All excited-state calculations were based on the geometry optimized at CAS $(10,8) / \mathrm{cc}$ pVDZ level.

The famous Rice-Ramsperger-Kassel-Marcus (RRKM) theory, based on the statistical equilibrium and harmonic assumption, has been proven to be an effective method to treat the rate constant of unimolecular reactions. The RRKM theory with tunneling effect was used to calculate rate constants of several reactions studied in this work. Ignoring rotational degrees of freedom, the expression for the unimolecular rate constant (units s ${ }^{-1}$ ) (RRKM) of an isolated molecule with total energy $E$ that incorporates tunneling is given by ${ }^{24}$

$$
k(E)=\frac{\sum_{n} P\left(E_{1}-\varepsilon_{n}^{\neq}\right)}{2 \pi \hbar N_{0}{ }^{\prime}(E)},
$$

where $\Sigma_{n} P\left(E_{1}-\varepsilon_{n}{ }^{\neq}\right)$is the integral density for the transition state with considering tunneling effect and $N_{0}(E)$ is the integral densities of states for the reactant molecule. Eckart potential model has been chosen to calculate the tunneling probability, which is considered as a good tunneling modification in general. It should be pointed out that ultrafast nonradiative processes occurring in 2-AP might reach the limitation of statistical equilibrium distribution, which is the premise for the transition-state rate calculations. Quantum dynamics in terms of quantum wave packets is more appropriate for describing ultrafast processes. However, quantum dynamics calculations require a very large amount of information about potential energy surfaces, which are hard or even impossible to obtain from first principles for the 2-AP molecule.

\section{RESULTS AND DISCUSSIONS}

\section{A. Fluorescent decay of 2-AP molecule}

The CASSCF/cc-pVDZ optimized geometry structures of $S_{0}$ and $S_{1}$ states are shown in Fig. 1. The $S_{0}$ equilibrium structure is nonplanar with two amino hydrogen atoms out of the plane of the aromatic ring, while the $S_{1}$ state has a nearly planar structure. At CAS $(10,8) / \mathrm{cc}-\mathrm{pVDZ}$ level, the $S_{1}$ state

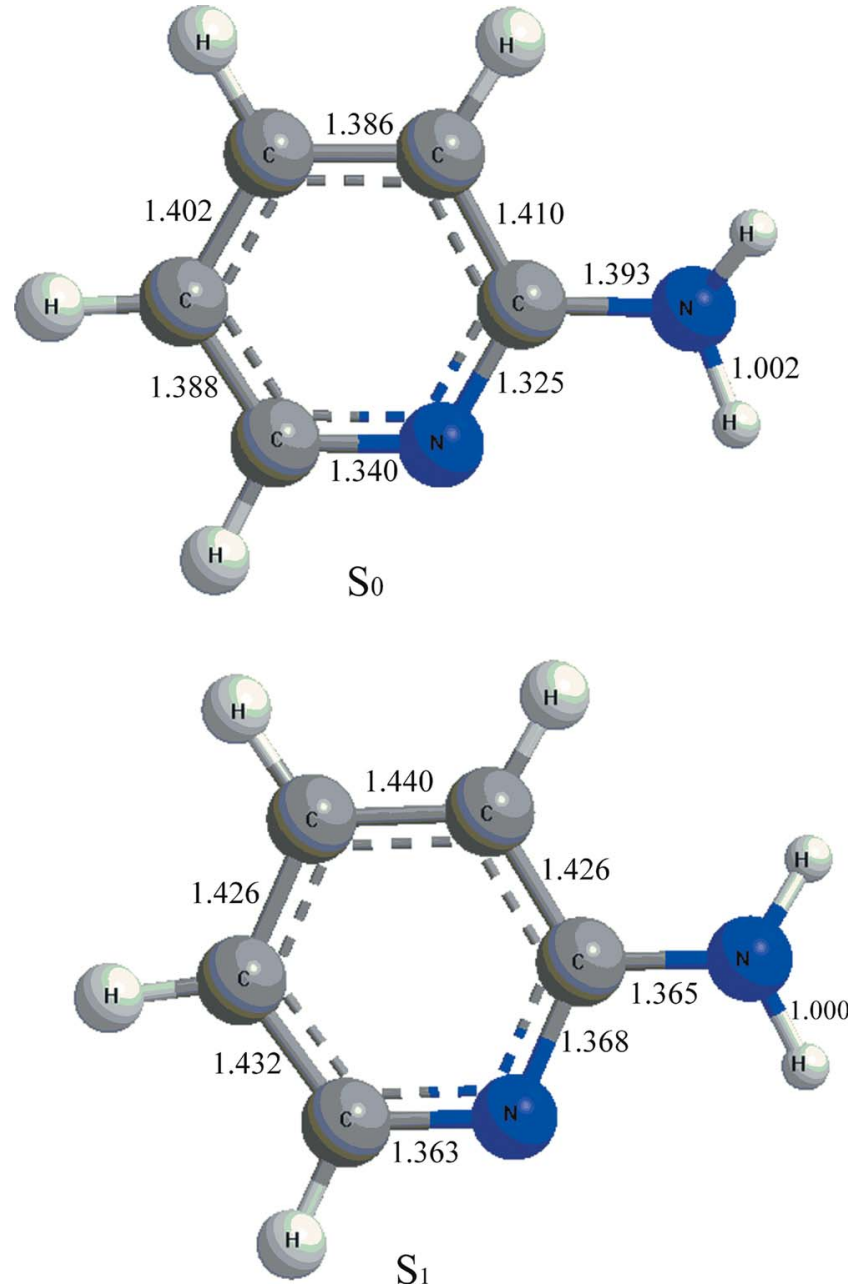

FIG. 1. (Color online) Optimized structures of ground state and first singlet excited states (unit of bond length: angstrom).

is $4.63 \mathrm{eV}$ higher than the ground state including the zero point energy, while the calculated vertical excitation energy is $5.03 \mathrm{eV}$.

Based on the equilibrium geometry of $S_{1}$ state optimized by CAS $(10,8) / c c-p V D Z$ method, emission energy and oscillator strength were calculated using LR at different theoretical levels, and the results are given in Table I. It could be seen that the MCSCF calculation gives much longer fluorescence lifetime than other methods. We have also calculated the absorption energy and oscillator strength based on the ground state geometry. In this case, LR and geometry opti-

TABLE I. Excitation energies, oscillator strength, and fluorescence lifetime calculated at different theoretical levels using cc-pVDZ basis set.

\begin{tabular}{|c|c|c|c|c|}
\hline & LR methods & $\begin{array}{c}\text { Excitation } \\
\text { energy } \\
\left(\mathrm{cm}^{-1}\right)\end{array}$ & Oscillator strength & $\begin{array}{c}\text { Fluorescence } \\
\text { lifetime } \\
\text { (ns) }\end{array}$ \\
\hline \multirow[t]{3}{*}{$S_{1}$} & $\operatorname{CAS}(10,8)^{\mathrm{a}}$ & 32858 & 0.037 & 37.71 \\
\hline & B3LYP ${ }^{a}$ & 35192 & 0.056 & 21.58 \\
\hline & $\mathrm{CCSD}^{\mathrm{a}}$ & 35420 & 0.059 & 19.69 \\
\hline \multirow[t]{3}{*}{$S_{0}$} & $\operatorname{MCSCF}(10,8)$ & 37361 & 0.032 & $\cdots$ \\
\hline & B3LYP & 38185 & 0.052 & $\cdots$ \\
\hline & CCSD & 39339 & 0.050 & $\cdots$ \\
\hline
\end{tabular}

${ }^{a}$ Geometry optimized at CAS(10,8) level. 


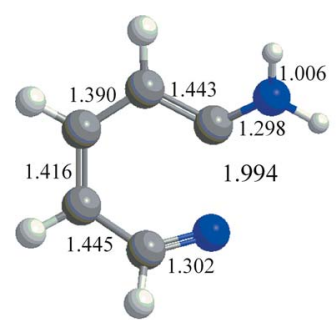

(a)

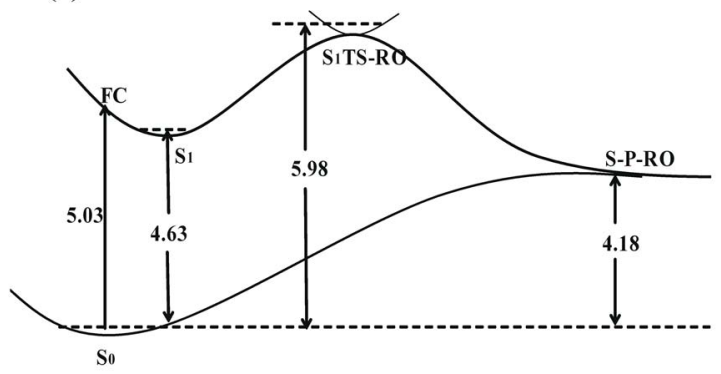

(c)

FIG. 2. (Color online) Optimized structures of transition state (a) and product (b) as well as energy profile (c) for the ring opening reaction (unit of energy: eV).

mization were carried out at the same theoretical level. The results for absorption process show the same methoddependence as that for the emission process, i.e., MCSCF LR calculation overestimates the oscillator strength. The most accurate result for fluorescence lifetime is from CCSD response calculation, which gives a value of $19.7 \mathrm{~ns}$. It is interesting to see that B3LYP result is very close to that of CCSD. Our calculations have at least led to one conclusion that the lifetime of $1.5 \mathrm{~ns}$ obtained in recent experiments ${ }^{1,11}$ for 2-AP molecule could not be solely from fluorescence. Certain nonradiative processes must be involved in the decay.

\section{B. Nonradiative photochemical pathways}

We have found four possible nonradiative photochemical pathways for the first excited state of 2-AP molecule, including ring opening, intramolecular hydrogen transfer, hydrogen detachment, and ring deformation reactions, whose mechanisms are discussed in detail below.

\section{Aromatic ring opening}

For such an aromatic ring system, an expected decay pathway could be aromatic ring opening reaction. ${ }^{25}$ The calculated stationary structures and potential energy profile are given in Fig. 2. The barrier for the ring opening on the $S_{1}$ state is $1.35 \mathrm{eV}$. If there should be enough energy to overcome the barrier, the aromatic ring could open at the $\mathrm{C}-\mathrm{N}$ bond, leading to a product with chainlike structure [Fig. 2(b)]. However, since the barrier is much higher than the vertical excitation energy of $5.03 \mathrm{eV}$, the probability for the ring opening could be very low.

\section{Intramolecular hydrogen transfer}

Hydrogen bonding between the hydrogen atom of amino group and the nitrogen atom on aromatic ring in 2-AP mol-

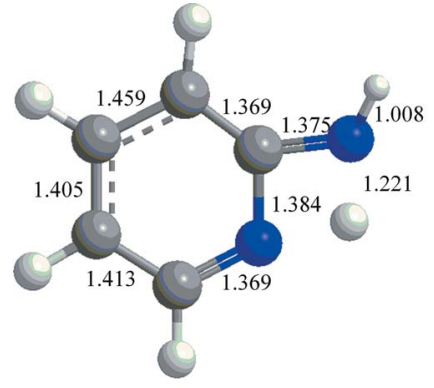

(a)

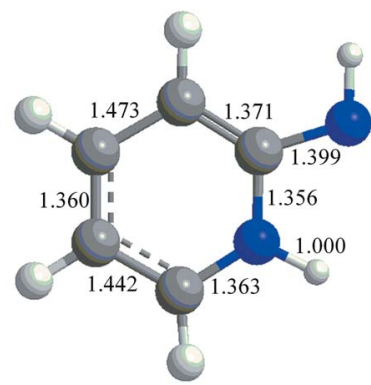

(b)

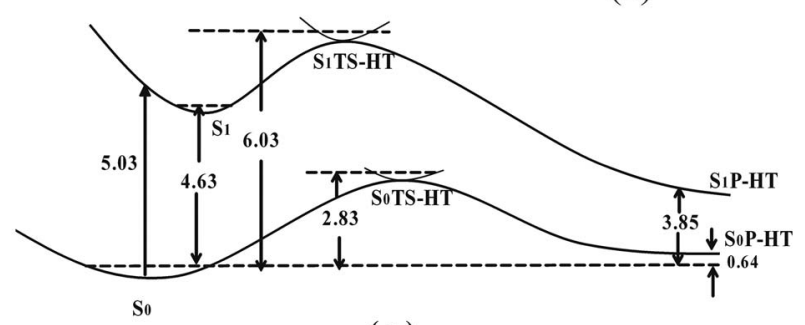

( c )

FIG. 3. (Color online) Optimized structures of transition state (a) and product (b) as well as energy profile (c) for the intramolecular hydrogen transfer reaction.

ecule was suggested by Hollas, ${ }^{14}$ which was also called as intramolecular hydrogen transfer. As a result, the $\pi$ character of the nitrogen atom on the aromatic ring is weakened, whereas the $\pi$ conjugated interaction between the amino group nitrogen and the aromatic ring becomes stronger. The structures of the transition state and product along this pathway, as well as the potential energy surface, are shown in Fig. 3. The transition state of this reaction is located about $6.03 \mathrm{eV}$ above the ground state, which is $1.00 \mathrm{eV}$ higher than the calculated vertical excitation energy. The calculated energy profile along the path of intramolecular hydrogen transfer as shown in Fig. 3(c) indicates that the energy of the ground state does not increase as much as that of the $S_{1}$ state. Therefore, the decay probability from $S_{1}$ to $S_{0}$ along this pathway could thus be very small.

\section{Hydrogen detachment}

There exists an alternative hydrogen transfer process for aromatic aminolike complex, namely, H-atom detachment, which was involved in an effective deactivation pathway for photoexcited pyrrole, indole, and phenol. ${ }^{26,27}$ For these systems, a repulsive ${ }^{1} \pi \sigma^{*}$ state intersects with the photoexcited ${ }^{1} \pi \pi^{*}$ state along the amino hydrogen detachment reaction pathway and decays to the ground state by a ultrafast internal conversion process via a conical intersection between the ${ }^{1} \pi \sigma^{*}$ state and the ground state. The $\mathrm{H}$-atom detachment mechanism of 2-AP molecule is also identified in this work; the two conical intersections of ${ }^{1} \pi \sigma^{*} /{ }^{1} \pi \pi^{*}$ and ${ }^{1} \pi \sigma^{*} / S_{0}$ are located along this pathway, as shown in Figs. 4(a) and 4(b). In Fig. 4(c), the energy of ${ }^{1} \pi \pi^{*}$ state is stepwise optimized as a function of $\mathrm{H}$-atom detachment coordinate with $\mathrm{Cs}$ symmetry constrain, and the energies of the ground state and ${ }^{1} \pi \sigma^{*}$ state are calculated at the optimized ${ }^{1} \pi \pi^{*}$ geometries. The energy of ${ }^{1} \pi \sigma^{*} /{ }^{1} \pi \pi^{*}$ conical intersection is found to be $1.65 \mathrm{eV}$ higher than the minimum $S_{1}$ state, which is even 


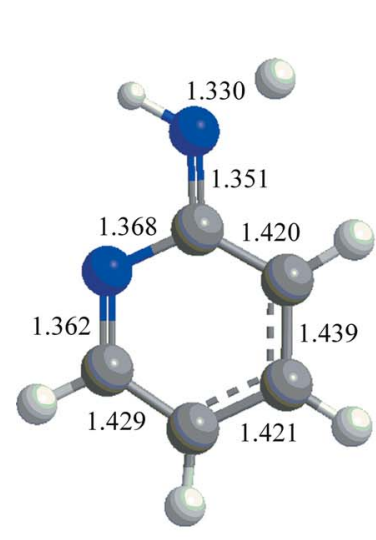

(a)

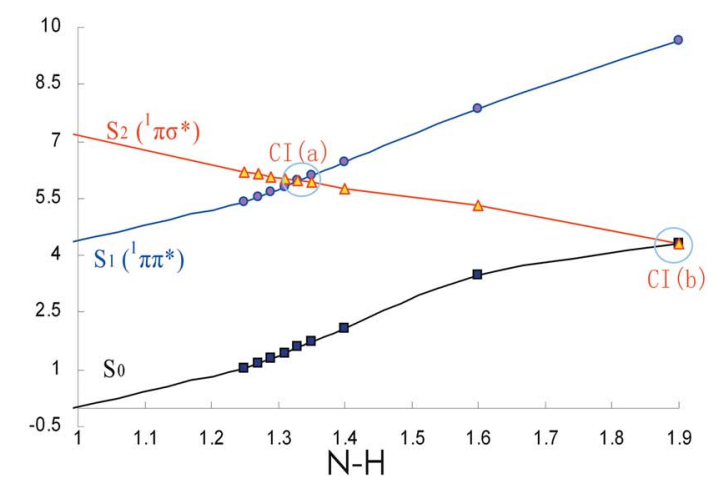

(c)

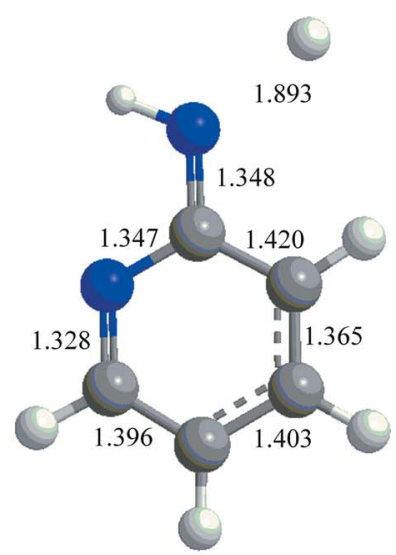

(b)
FIG. 4. (Color online) Structures of the ${ }^{1} \pi \sigma^{*} /{ }^{1} \pi \pi^{*}\left(S_{1} / S_{2}\right)$ (a) and ${ }^{1} \pi \sigma^{*} / S_{0}$ $\left(S_{2} / S_{0}\right)$ (b) as well as the energy curve (c) for the H-atom detachment reaction.

higher than the barrier of intramolecular hydrogen transfer reaction $(1.40 \mathrm{eV})$ as given in Fig. 3. Therefore for 2-AP system, the $\mathrm{H}$-atom detachment reaction pathway could not provide an effective deactivation pathway as it did for other molecules, ${ }^{26,27}$ even though the energy of the ${ }^{1} \pi \sigma^{*} / S_{0}$ conical intersection is quite low.

\section{Ring deformation pathway and $S_{1} / S_{0}$ conical intersection}

A prefulvenic reaction pathway known as "channel three effect" was suggested for benzene and benzenelike molecules, ${ }^{28-30}$ which corresponded to a fast nonradiative deactivation of excited state. One carbon atom in the aromatic ring tends to move out of the ring during this reaction pathway. Experimental and theoretical studies for the ${ }^{1} \pi \pi^{*}$ state deactivation of the pyridine molecule have led to the conclusion that along the prefulvenic pathway, there was an ultrashort internal conversion to the ground state. ${ }^{20}$ In this work, a similar reaction pathway, named as ring deformation reaction, is found for the ${ }^{1} \pi \pi^{*}$ state of 2 -AP molecule. A transition state with a barrier height of $0.13 \mathrm{eV}$ is identified by frequency analysis, which has a prefulvenelike structure. We have located $S_{1} / S_{0}$ conical intersection and its optimized structure is shown in Fig. 5(b). It is found that the molecular structure at conical intersection is quite close to the transition state [Fig. 5(a)]. A better picture to illustrate the prefulvenic

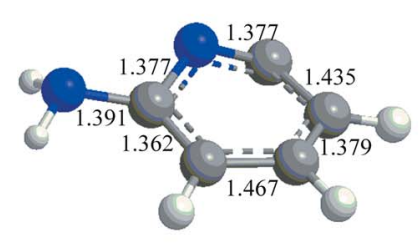

(a)

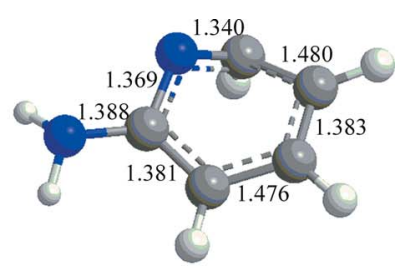

(b)

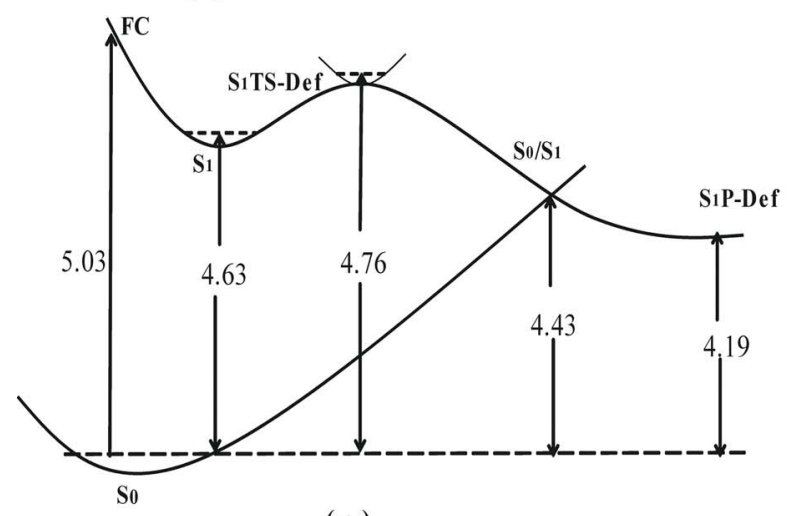

(c)

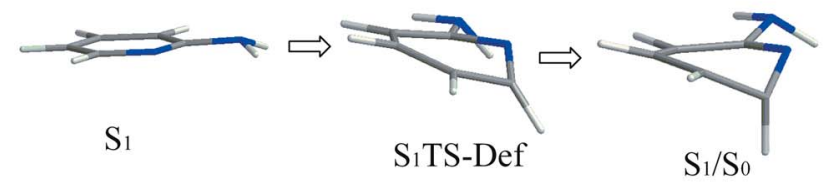

(d)

FIG. 5. (Color online) Optimized structure of the transition state (a) and $S_{0} / S_{1}$ conical interaction as well as the energy profile (c) for the ring deformation reaction.

process is given in Fig. 5(d). From the energy potential profile in Fig. 5(c), one can see that resonant vertical excitation with energy of $5.03 \mathrm{eV}$ is adequate to overcome the low energy barrier and reaching the ground state through the conical intersection. In another word, this is a rapid pathway for the decay of the $S_{1}$ state involving internal conversion process.

\section{Rate constants for various nonradiative decay pathways}

We have employed RRKM theory with inclusion of tunneling effect to calculate rate constants of the proposed three photochemical reactions (ring opening, intramolecular hydrogen transfer, and the ring deformation); the results are given in Table II. For the hydrogen detachment reaction, the rate constant was not calculated because of its high energy barrier. Apparently both intramolecular hydrogen transfer

TABLE II. Rate constants of three reaction pathways calculated with CAS(10,8)/cc-pVDZ.

\begin{tabular}{lc}
\hline \hline Reaction pathways & $\begin{array}{c}\text { Rate constant } \\
\left(\mathrm{s}^{-1}\right)\end{array}$ \\
\hline Hydrogen transfer & $1.79 \times 10^{-5}$ \\
Ring opening & $1.57 \times 10^{-37}$ \\
Ring deformation & $8.69 \times 10^{11}\left(1.17 \times 10^{10}\right)^{\mathrm{a}}$ \\
\hline \hline
\end{tabular}

${ }^{\mathrm{a}}$ Excitation energy from CCSD LR calculation. 
and ring opening reactions could not compete with the ring deformation reaction, whose rate on the $S_{1}$ state is found to be $8.69 \times 10^{11} \mathrm{~s}^{-1}$ at $\operatorname{CAS}(10,8)$ level. Such a rate corresponds to a very short lifetime of $1.1 \mathrm{ps,} \mathrm{which} \mathrm{is} \mathrm{much}$ lower than the fluorescence lifetime of $19.7 \mathrm{~ns}$. One thing seems to be clear that photochemical property of 2-AP molecule is similar to that of pyridine and DNA bases, ${ }^{20,22}$ for which the nonradiative process (internal conversion via the $S_{1} / S_{0}$ conical intersection) also dominates the whole decay processes. The combination of both radiative ad nonradiative lifetimes leads to a value of $3.3 \mathrm{ps,} \mathrm{which} \mathrm{is} \mathrm{much} \mathrm{smaller}$ than the experimental value of $1.5 \mathrm{~ns}$. It is noted that the nonradiative lifetime is sensitive to the barrier height for the ring deformation reaction and the vertical excitation energy. For instance, when the CCSD result $(4.88 \mathrm{eV})$ for the vertical excitation energy is used, the lifetime could become 8.5 ns. It is very unfortunate that limited by our computational capacity, it is not possible to obtain the entire potential surface from higher-level calculations. In addition, the theoretical model used here could overestimate the rate of radiationless decay of 2-AP molecule.

\section{CONCLUSIONS}

Our calculations present an ultrashort nonradiative process for first excited 2-AP molecule involving a ring deformation reaction pathway on $S_{1}$ state. The presence of low reaction energy barrier and conical intersection between $S_{1}$ and $S_{0}$ states along this pathway could probably result in a fast decay rate of excited 2-AP. The theoretically fluorescence lifetime for 2-AP molecule could not compete with the fast nonradiative deactivation. We have shown that the lifetime of the $S_{1}$ state of 2-AP molecule is very sensitive to the barrier height along the reaction path of the ring deformation and consequently is sensitive to the level of theory.

\section{ACKNOWLEDGMENTS} (VR).
${ }^{1}$ T. Schultz, E. Samoylova, W. Radloff, I. V. Hertel, A. L. Sobolewski, and W. Domcke, Science 306, 1765 (2004).

${ }^{2}$ A. L. Sobolewski and W. Domcke, Chem. Phys. 294, 73 (2003).

${ }^{3}$ A. L. Sobolewski and W. Domcke, Phys. Chem. Chem. Phys. 6, 2763 (2004).

${ }^{4}$ A. L. Sobolewski, W. Domcke, and C. Hattig, Proc. Natl. Acad. Sci. U.S.A. 102, 17903 (2005)

${ }_{5}^{5}$ A. Abo-Riziq, L. Grace, E. Nir, M. Kabelac, P. Hobza, and M. S. Vries, Proc. Natl. Acad. Sci. U.S.A. 102, 20 (2005).

${ }^{6}$ J. R. Roscioli and D. W. Pratt, Proc. Natl. Acad. Sci. U.S.A. 100, 13752 (2003).

${ }^{7}$ C. E. Crespo-Hernandez, B. Cohen, and B. Kohler, Nature (London) 436, 1141 (2005).

${ }^{8}$ C. E. Crespo-Hernandez, B. Cohen, P. M. Hare, and B. Kohler, Chem. Rev. (Washington, D.C.) 104, 1977 (2004).

${ }^{9}$ H. Kang, K. T. Lee, B. Jun, and J. K. Kim, J. Am. Chem. Soc. 124, 12598 (2002).

${ }^{10}$ H. Satzger, D. Townsend, M. Z. Zgierski, S. Patchkovski, S. Ullrich, and A. Stolow, Proc. Natl. Acad. Sci. U.S.A. 103, 10196 (2006).

${ }^{11}$ D. R. Borst, J. R. Roscioli, and D. W. Pratt, J. Phys. Chem. A 106, 4022 (2002).

${ }^{12}$ S. Babiak and A. C. Testa, J. Phys. Chem. 80, 1882 (1976).

${ }_{13}^{13}$ A. C. Testa and U. P. Wild, J. Phys. Chem. 83, 3044 (1979).

${ }^{14}$ J. M. Hollas, H. Musa, and T. Ridley, J. Mol. Spectrosc. 104, 89 (1984).

${ }^{15}$ J. Hager and S. C. Wallace, J. Phys. Chem. 89, 3833 (1985).

${ }^{16}$ J.-M. L. Pecourt, J. Peon, and B. Kohler, J. Am. Chem. Soc. 123, 10370 (2001).

${ }^{17}$ N. Ismail, L. Blancafort, M. Olivucci, B. Kohler, and M. Robb, J. Am. Chem. Soc. 124, 6818 (2002).

${ }^{18}$ M. Merchan and L. Serrano-Andres, J. Am. Chem. Soc. 125, 8108 (2003).

${ }^{19}$ S. Perun, A. L. Sobolewski, and W. Domcke, J. Am. Chem. Soc. 127, 6257 (2005).

${ }^{20}$ M. Chachisvilis and A. H. Zewail, J. Phys. Chem. A 103, 7408 (1999).

${ }^{21}$ M. Barbatti and H. Lischka, J. Phys. Chem. A 111, 2852 (2007).

${ }^{22}$ M. J. Frisch, G. W. Trucks, H. B. Schlegel et al., GAussian 03, Revision C.02, Gaussian, Inc., Wallingford, CT, 2004.

${ }^{23}$ DALTON, Release 2.0, a molecular electronic structure program (2005) (http://www.kjemi.uio.no/software/dalton/dalton.html).

${ }^{24}$ W. H. Miller, J. Am. Chem. Soc. 101, 6810 (1979).

${ }^{25}$ M. Z. Zgierski, S. Patchkovskii, and E. C. Lim, J. Chem. Phys. 123, 081101 (2005).

${ }^{26}$ M. N. R. Ashfold, B. Cronin, A. L. Devine, R. N. Dixon, and M. G. D. Nix, Science 312, 1637 (2006).

${ }^{27}$ O. P. J. Vieuxmaire, Z. Lan, A. L. Sobolewski, and W. Domcke, J. Chem. Phys. 129, 224307 (2008).

${ }^{28}$ G. R. Unruh and D. M. Birney, J. Am. Chem. Soc. 125, 8529 (2003).

${ }^{29}$ S. Kato, J. Chem. Phys. 88, 3045 (1988).

${ }^{30}$ I. J. Palmer, I. N. Ragazos, F. Bernardi, M. Olivucci, and M. A. Robb, J. Am. Chem. Soc. 115, 673 (1993). 